\title{
Estimating the size of an earthquake using short-period seismograms of the first three seconds: A simulated experiment using the 1999 Chi-Chi earthquake sequence*
}

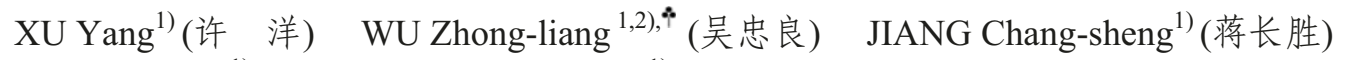 \\ YU Yan-xiang ${ }^{1)}$ (俞言祥) YANG Jian-si ${ }^{1)}$ (杨建思) \\ 1) Institute of Geophysics, China Earthquake Administration, Beijing 100081, China \\ 2) College of Earth Science, Graduate University of Chinese Academy of Sciences, Beijing 100049, China
}

\begin{abstract}
Most of the present earthquake early warning systems are based on broadband or strong motion recordings. However, the short-period instruments are still deployed. It is well-known that short-period recordings have saturation problems for large earthquakes when estimating the size of an earthquake. Thus, it is necessary to make clear the magnitude at which saturation starts to occur for the commonly used $\tau_{c}$ and $P d$ measurements, respectively. To investigate the possibility of using short-period seismic recordings for earthquake early warning, we conducted a simulated experiment using the strong motion data of the 1999 Chi-Chi earthquake sequence including its main shock and 31 aftershocks, with magnitude span from 4 to 7.6. The strong motion acceleration recordings were convolved with the instrument response of short-period seismographs in northern China to simulate short-period seismograms. Parameters $\tau_{c}$ and $P d$ from the first-three-second seismograms were calculated for the simulated short-period recordings and compared with that obtained by the original strong ground motion recordings. The result showed that to some extent, short-period recordings can be used for threshold earthquake early warning, while the magnitude saturation of $P d$ estimation can be up to 6.5 , better than $\tau_{c}$ estimation.
\end{abstract}

Key words: earthquake early warning system; $\boldsymbol{\tau}_{c}$ measurement; $\boldsymbol{P} \boldsymbol{d}$ measurement; short-period recording CLC number: P315.8 Document code: A

\section{Introduction}

Over the past few years, research and development of different types of earthquake early warning systems (EEWS) have shown significant potential for the mitigation of earthquake disasters (Heaton, 1985; Nakamura, 1988, 1989; Espinosa-Aranda et al, 1995; Kanamori et al, 1997; Wu and Teng, 2002; Allen and Kanamori, 2003; Kanamori, 2005; Wu and Kanamori, 2005a, b; 2007; Olson and Allen, 2005; Horiuchi et al, 2005; Zollo et al, 2006; Boese et al, 2007), among which a challenging type of EEW is the on-site earthquake early warning. One of the critical

\footnotetext{
Received 2007-09-11; accepted in revised form 2008-01-21.

Foundation item: Foundation for Seismological Researches.

Contribution No. 08FE3003, Institute of Geophysics, China Earthquake Administration.

^Author for correspondence: wuzhl@gucas.ac.cn
} 
points of on-site EEWS is to determine the overall size of an earthquake, and thus the expected strong ground motion, from the first few seconds of the $\mathrm{P}$ wave (Wu et al, 2006; Wu and Zhao, 2006). Olson and Allen (2005) suggested that the final magnitude of an earthquake could be determined by the first few seconds of $\mathrm{P}$ wave onset in the broadband seismograms, which reflects the rupture process and the stress state in the source region, providing a physical basis for the EEWS applications. Although the controversy in physics of seismic source exists (Rydelek and Horiuchi, 2006), such an observation is valid for earthquakes with magnitude lower than 6.0. For higher magnitudes, more researches are still needed to investigate whether the relation between the first-three-second properties and the final magnitude of the earthquake still holds.

When the physics and system of on-site EEW are applied to most of the regions in the world other than the well-equipped areas such as Japan, Taiwan, and California, there is a special difficulty that most of the stations deployed are not broadband but short-period. The recordings of the short-period stations have been used for a long time mainly for the monitoring of local seismic activity. Whether these short-period seismic recordings could be used in the earthquake early warning purpose is a problem which is not only interesting in theory but also practical in application. It is well-known that one of the critical problems for short-period recordings to be used in estimating the size of an earthquake is saturation. Practically in the EEWS, however, it is necessary to make clear when using $\tau_{c}$ and $P d$ measurements, where the saturation starts to occur (Kanamori, 2005; Wu and Kanamori, 2005a; Wu et al, 2006). To investigate this problem we used the main shock and aftershock recordings of the 1999 Chi-Chi earthquake and conduct a simulated experiment. The result showed that "the first three second approach", that is, using the seismograms of the first three seconds of $\mathrm{P}$ wave seismograms to estimate the size of an earthquake, can be used to short-period seismic recordings to some extent, while the $P d$ estimation seems better than the $\tau_{c}$ estimation with a higher saturation magnitude.

\section{$1 \tau_{c}$ and $P d$ measurement}

Following previous studies (Kanamori, 2005; Wu and Kanamori, 2005a; Wu et al, 2006, 2007), we took the $\tau_{c}$ and $P d$ approaches in our simulated experiment. In the $\tau_{c}$ approach, ground motion displacement $u(t)$ and velocity $\dot{u}(t)$ from the vertical component seismograms were taken. Then the ratio $r$

$$
r=\frac{\int_{0}^{\tau_{0}} \dot{u}^{2}(t) \mathrm{d} t}{\int_{0}^{\tau_{0}} u^{2}(t) \mathrm{d} t}
$$

was computed, where the integration was taken over the time interval $\left(0, \tau_{0}\right)$ after the onset of the $\mathrm{P}$ wave. Usually, $\tau_{0}$ is selected as $3 \mathrm{~s}$ (Kanamori, 2005). Parseval's theorem tells that

$$
r=\frac{4 \pi^{2} \int_{0}^{\infty} f^{2}|\hat{u}(f)|^{2} \mathrm{~d} f}{\int_{0}^{\infty}|\hat{u}(f)|^{2} \mathrm{~d} f}=4 \pi^{2}<f^{2}>
$$

where $\hat{u}(f)$ is the spectrum of $u(t)$, and $<f^{2}>$ is the average of $f^{2}$ weighted by $|\hat{u}(f)|^{2}$. Based on these spectra, parameter $\tau_{c}$ is defined by (Wu and Kanamori, 2005a) 


$$
\tau_{c}=\frac{1}{\sqrt{\left\langle f^{2}\right\rangle}}=\frac{2 \pi}{\sqrt{r}}
$$

In physics, it can be interpreted as a parameter representing the characteristic period of the initial portion of the $\mathrm{P}$ wave. Empirical relation gives that the larger $\tau_{c}$ is, the larger the event size will be: Previous study showed that the values of $\log \tau_{c}$ increase with the earthquake magnitude $M$ (Wu et al, 2006, 2007), via

$$
M_{\tau_{c}}=a \log \tau_{c}+b
$$

where $a$ and $b$ are constants, and $M_{\tau_{c}}$ is the estimated magnitude using $\tau_{c}$ measurement.

The quantity $P d$ is defined by the peak amplitude of displacement within the first few seconds (again usually $3 \mathrm{~s}$ ) after the arrival of the $\mathrm{P}$ wave (Wu et al, 2006). An approximate linear relationship among the logarithmic $P d$, the estimated magnitude $M_{P d}$, and the logarithmic hypocentral distance $R$ can be represented by

$$
M_{P d}=A \log (P d)+B \log (R)+C
$$

where $A, B$, and $C$ are empirical constants. Therefore, if the attenuation relationship of $P d$ is determined, then $P d$ can be used to estimate the magnitude when the hypocentral distance is available. For short hypocentral distances, i.e., for the case of on-site EEW, one has the simplified version

$$
M_{P d}=A^{\prime} \log (P d)+C^{\prime}
$$

in which $A^{\prime}$ and $C^{\prime}$ are constants. We calculated $\tau_{c}$ and $P d$ using the tools of MatLab.

\section{Data analysis}

The existing relationships between the magnitudes of earthquakes and some observational properties of the first few seconds of $\mathrm{P}$ wave, including $\tau_{c}$ and $P d$ (Kanamori, 2005; Wu and Kanamori, 2005a, b; Wu et al, 2006), are mainly for broadband recordings. In this study, however, our attention has to be paid to short-period seismograms. The approach is straightforward: to compare the results obtained by simulated short-period seismograms and original strong motion seismograms. Intuitively there should be saturation for large events, but where the saturation occurs is a question we have to answer by investigating real seismic data.

We used the Central Weather Bureau (CWB) Free-Field Strong-Motion Data of the 1999 Chi-Chi earthquake (Lee et al, 2001) for the simulated experiment. Totally 34 events (the main shock and 33 aftershocks) are included in this data set. We selected 32 events with $M>4.0$ and focal depth $<25 \mathrm{~km}$ for this study (as listed in Table 1). The other two events were discarded due to low signal-to-noise ratio. All the chosen events were well recorded by the Taiwan Strong Motion Instrumentation Program (TSMIP) network which includes about 650 digital accelerographs installed at free-field sites. The strong motion instruments have a flat response from DC to $50 \mathrm{~Hz}^{(1)}$ (Chen et al, 2006; Wu et al, 2001) and a full-scale range of $\pm 2 \mathrm{~g}$. The 16-bit outputs were digitized at 200 or 250 samples per second. Figure 1 shows the chosen events from the 1999 Chi-Chi earthquake sequence as well as the location of the strong motion accelerators whose recordings were used in this study. In previous studies, a number of the nearest recordings of each event were

\footnotetext{
(1) Kinemetrics Inc. 1997. User's Guider-The FBA-23 Force Balance Accelerometer.
} 


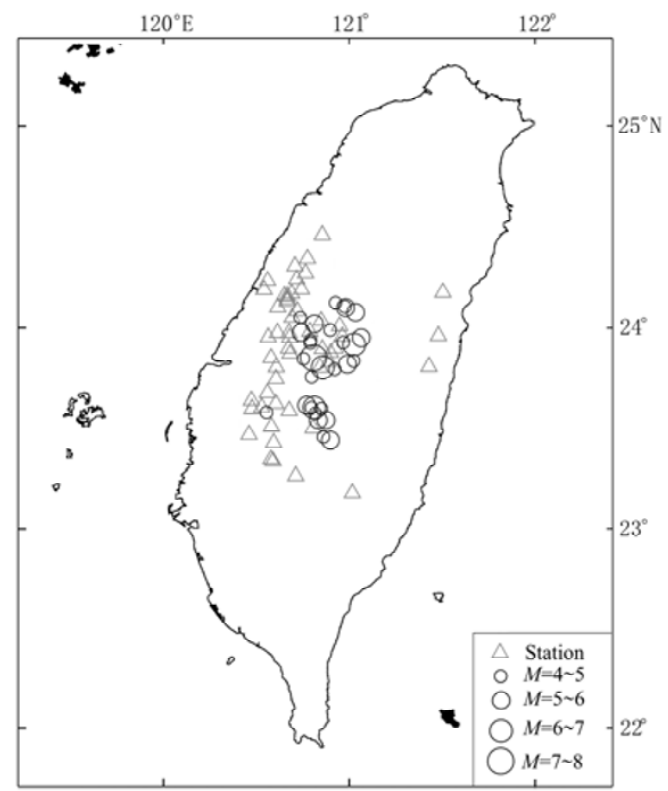

Figure 1 Stations (triangles) of the Taiwan Strong Motion Instrumentation Program (TSMIP) and the epicenters of the 32 earthquakes (circles) used in this study calculated to determine parameters $\tau_{c}$ and $P d$. Similarly, in our experiment, we chose the closest eight station's recordings for each event, and used the average value for the eight stations for the magnitude estimation. In this case, most of recordings were chosen at epicentral distances less than $35 \mathrm{~km}$, while few recordings have epicentral distances up to 52 $\mathrm{km}$. Vertical component recordings were used in this experiment. Acceleration was integrated once to obtain velocity and integrated twice to obtain displacement. A $0.075 \mathrm{~Hz}$ Butterworth high-pass filter was applied to remove the low-frequency drift after the integration. We used an automatic $\mathrm{P}$ picker similar to that described by Allen (1978) to detect the $\mathrm{P}$ wave arrival. We then computed $\tau_{c}$ from the first 3-second-long filtered signals after the $\mathrm{P}$ arrival. The peak displacement amplitude $P d$ was also computed for the same time window.

To simulate short-period seismograms, the strong motion acceleration recordings were convolved with the instrument response of short-period seismographs in North China (Institute of Geophysics, China Earthquake Administration, 2006). The instrument response of Taiwan strong motion accelerator is flat in the whole frequency band (DC to $50 \mathrm{~Hz}$ ), thus the strong motion acceleration recordings can be treated approximately as original strong ground motion. The integral over time of such a convolved time-series gives the short-period velocity recordings as seen in the seismograms of the North China Seismic Network. Reversely, direct convolution of the strong motion recordings with the short-period instrument response corresponds to the differentiation of the short-period seismograms, or in another word, gives the simulated "short-period acceleration recordings". Figure 2 shows the comparison between the strong motion acceleration recording and the simulated short-period acceleration recording of the Chi-Chi main shock. From the figure it can be seen that for the first few seconds after the P-arrival, especially for the first three seconds, the strong motion recording and the simulated short-period one are similar to each other, providing us with sufficient confidence of using short-period recordings in EEW. Parameters $\tau_{c}$ and $P d$ from the first 3-second-long seismograms were calculated for the simulated short-period seismograms and then compared with that obtained directly from the strong motion recordings.

\section{Results}

For all the aftershocks and the main shock, we use the magnitude originally provided by CWB. In the CWB report, $M_{\mathrm{L}}$ measurement is used for aftershocks, and the Harvard moment magnitude is used for the main shock. Similar to previous studies (Wu et al, 2006), in Table 1, we 
Table 1 Events used in this study

\begin{tabular}{|c|c|c|c|c|c|c|}
\hline No. & $\begin{array}{c}\text { Date } \\
\text { a-mo-d }\end{array}$ & $\begin{array}{c}\text { Origin time (UTC) } \\
\text { h:min:s }\end{array}$ & $\varphi_{\mathrm{N}} /^{\circ}$ & $\lambda_{\mathrm{E}} /^{\circ}$ & Depth $/ \mathrm{km}$ & $M$ \\
\hline 1 & 1999-09-20 & $17: 57: 15.3$ & 23.93 & 121.03 & 11 & 6.4 \\
\hline 2 & 1999-09-20 & $18: 03: 41.2$ & 23.81 & 120.86 & 8 & 6.6 \\
\hline 3 & 1999-09-20 & $18: 21: 28.6$ & 23.96 & 121.06 & 10 & 5.2 \\
\hline 4 & $1999-09-20$ & $18: 32: 55.1$ & 23.83 & 120.99 & 17 & 5.1 \\
\hline 5 & 1999-09-20 & $18: 34: 25.9$ & 23.84 & 121.02 & 21 & 4.9 \\
\hline 6 & 1999-09-20 & $18: 50: 49.3$ & 23.93 & 120.97 & 14 & 4.8 \\
\hline 7 & 1999-09-20 & $18: 56: 04.5$ & 23.77 & 120.80 & 9 & 4.7 \\
\hline 8 & $1999-09-20$ & $19: 17: 10.7$ & 23.93 & 120.79 & 11 & 4.4 \\
\hline 9 & 1999-09-20 & $19: 19: 49.6$ & 24.00 & 120.90 & 19 & 4.6 \\
\hline 10 & 1999-09-20 & $19: 34: 51.0$ & 23.85 & 120.76 & 10 & 4.7 \\
\hline 11 & $1999-09-20$ & $19: 40: 32.6$ & 23.55 & 120.88 & 7 & 5.3 \\
\hline 12 & $1999-09-20$ & $19: 44: 56.3$ & 24.06 & 120.74 & 9 & 4.6 \\
\hline 13 & $1999-09-20$ & $19: 57: 52.6$ & 24.03 & 120.81 & 12 & 5.2 \\
\hline 14 & 1999-09-20 & $20: 02: 15.9$ & 23.98 & 120.74 & 12 & 5.4 \\
\hline 15 & 1999-09-20 & $20: 08: 11.4$ & 24.13 & 120.93 & 11 & 4.8 \\
\hline 16 & 1999-09-20 & $20: 11: 39.5$ & 23.95 & 120.80 & 10 & 4.5 \\
\hline 17 & 1999-09-20 & $20: 21: 59.7$ & 24.11 & 120.98 & 11 & 5.2 \\
\hline 18 & 1999-09-20 & $20: 29: 20.9$ & 23.80 & 120.92 & 15 & 4.8 \\
\hline 19 & 1999-09-20 & $21: 23: 23.9$ & 23.61 & 120.85 & 10 & 4.9 \\
\hline 20 & 1999-09-20 & $21: 27: 56.7$ & 24.09 & 121.03 & 12 & 5.0 \\
\hline 21 & 1999-09-20 & $21: 39: 53.7$ & 23.59 & 120.56 & 14 & 4.1 \\
\hline 22 & $1999-09-20$ & $21: 46: 37.5$ & 23.61 & 120.81 & 1 & 6.6 \\
\hline 23 & 1999-09-20 & $21: 54: 47.1$ & 23.62 & 120.77 & 4 & 5.3 \\
\hline 24 & $1999-0920$ & $21: 57: 12.4$ & 23.47 & 120.86 & 9 & 4.7 \\
\hline 25 & 1999-09-20 & $22: 15: 34.0$ & 23.59 & 120.82 & 3 & 4.7 \\
\hline 26 & $1999-09-20$ & $22: 22: 46.0$ & 23.55 & 120.83 & 5 & 5.2 \\
\hline 27 & 1999-09-20 & $22: 33: 54.8$ & 24.11 & 120.98 & 13 & 4.8 \\
\hline 28 & 1999-09-20 & $22: 56: 45.9$ & 23.88 & 120.92 & 12 & 4.9 \\
\hline 29 & 1999-09-20 & $22: 58: 52.5$ & 23.61 & 120.80 & 11 & 4.6 \\
\hline 30 & 1999-09-20 & $23: 18: 13.2$ & 23.45 & 120.91 & 10 & 5.1 \\
\hline 31 & $1999-09-22$ & $00: 14: 40.8$ & 23.83 & 121.05 & 16 & 6.8 \\
\hline 32 & 1999-09-20 & $17: 46: 40.0$ & 23.86 & 120.81 & 11 & 7.6 \\
\hline
\end{tabular}

denote all the magnitudes simply by $M$. It is clear that the saturation problem discussed in this paper is different from what we discussed in a general sense. Here when we say "saturation", we mean the underestimation of magnitude by using short-period seismograms compared to the magnitude estimated by strong motion recordings, no matter which type of magnitude is used.

Figure 3a shows the relationship between $M_{\tau_{c}}$ determined from the first three second $\mathrm{P}$ wave seismograms recorded by strong motion instruments versus the final magnitude $M$ of the earthquake (reported by CWB). The values of $M_{\tau_{c}}$ increase approximately linearly with $M$ with a linear correlation coefficient 0.86 and a standard deviation 0.41 . As for simulated short-period recordings, Figure $3 \mathrm{~b}$ shows that the correlation coefficient decreases to 0.53 and the standard deviation increases to 0.67 . The poor linear regression is mainly due to the saturation for $M>6$ events. Magnitude 6 for the position of saturation is to much extent a rough estimation because of the lack of data from magnitude 5.5 to 6.3 . The crossover of the linearly increasing part of the magnitudes and the saturation part gives that the saturation is approximately at 6.0.

Figure 4a shows the relationship between estimated $M_{P d}$ from strong motion recordings and the final magnitude $M$. The values of $M_{P d}$ increase linearly with $M$. Only the $M_{\mathrm{W}} 7.6$ Chi-Chi earthquake violates the relationship and has a much lower magnitude estimated by $P d$. Evidently this underestimation is not due to the choice of different types of magnitudes: If the magnitude were changed to $M_{\mathrm{L}}$, such underestimation would be more severe. For the simulated short-period 
recordings shown in Figure $4 \mathrm{~b}$, saturation occurs for events above magnitude 6.5. Comparing with the case of strong motion recordings, the correlation coefficient decreases slightly from 0.93 to 0.85 , while the standard deviation increases from 0.29 to 0.42 .

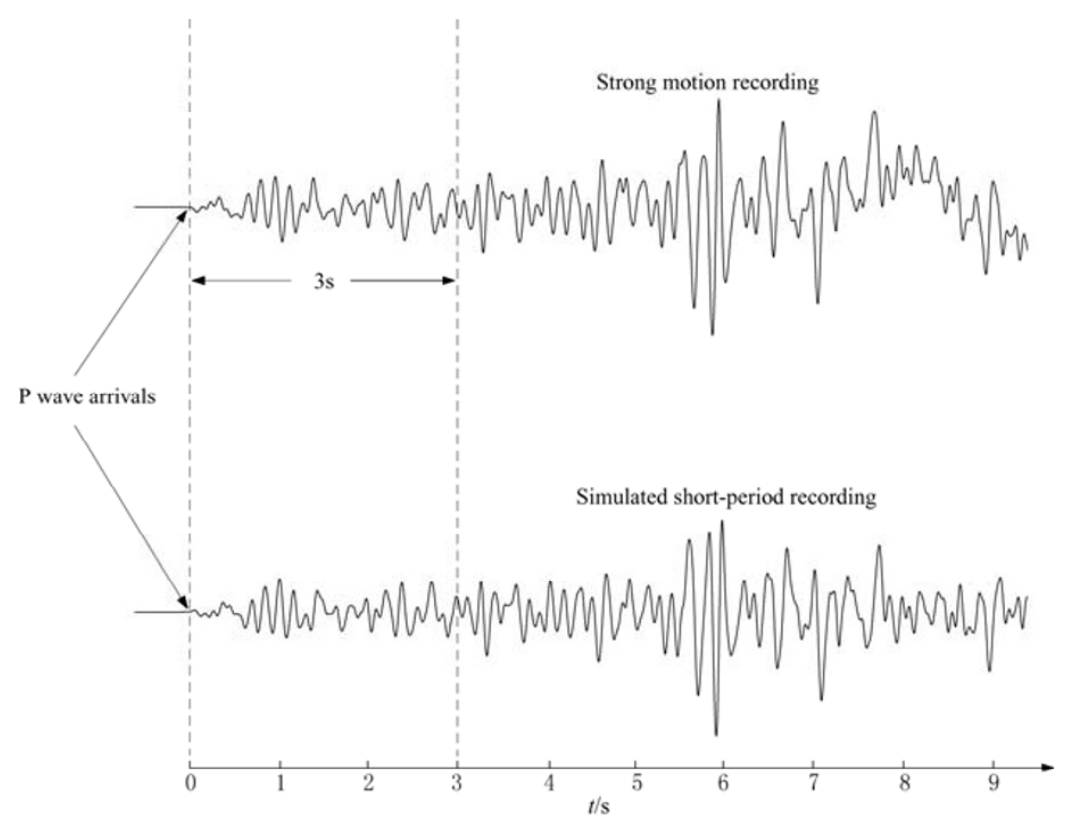

Figure 2 Vertical component strong motion (acceleration) recording and the simulated short-period acceleration recordings of the 1999 Chi-Chi main shock

The portion of the first three seconds is indicated by the two vertical dashed lines. Note that the short-period (SP) seismographs record velocities rather than accelerations. Therefore the bottom trace corresponds to the differentiation of the SP seismogram seen in the seismological network
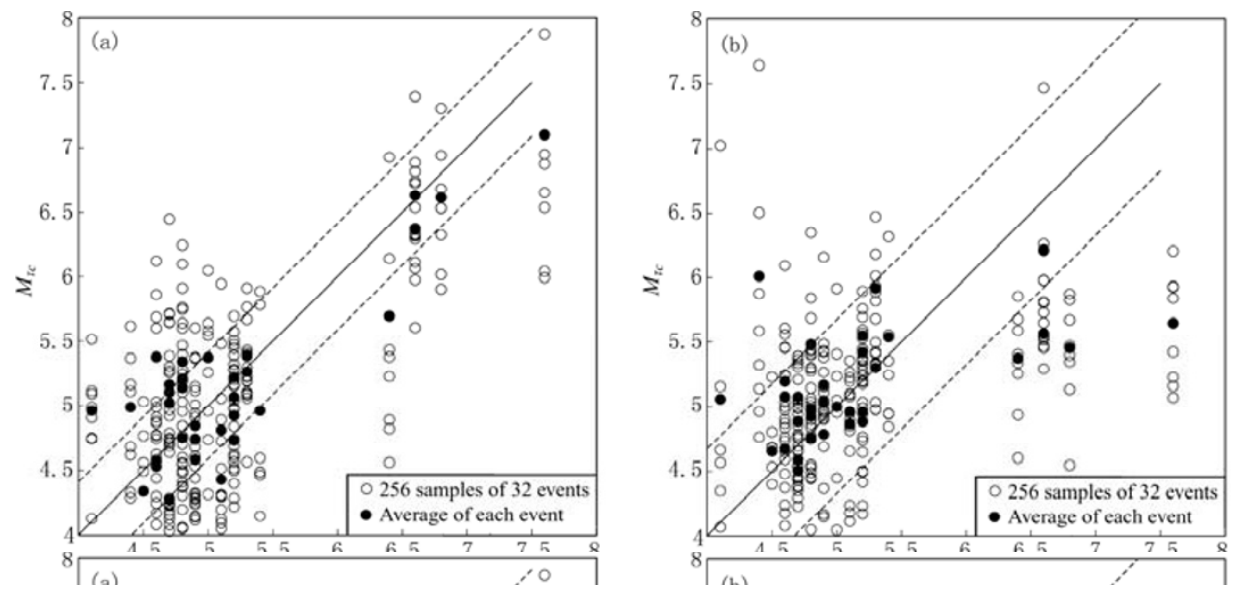

Figure 3 Magnitude estimated from $\tau_{c}$ calculated using strong motion recordings (a) and using simulated short-period recordings (b) (vertical axis) versus the magnitude of the earthquake (horizontal axis)

Solid line shows the least squares fit and two dashed lines show the range of one standard deviation. In Figure 3a standard deviation is 0.41 and correlation coefficient is 0.86 , and in Figure $3 \mathrm{~b}$ standard deviation is 0.67 and correlation coefficient is 0.53 
Figure 5a shows the comparison of the $\tau_{c}$-magnitude $M_{\tau_{c}}$ obtained from strong motion recordings with that from the simulated short-period recordings. The $45^{\circ}$ line is the theoretical line for $M_{\tau_{c}}(\mathrm{SM})=M_{\tau_{c}}(\mathrm{SP})$. Downward deviation from this line shows the saturation. From the figure it can be seen that apparent saturation occurs for magnitudes greater than 6 . The saturation magnitude is higher in the $P d$ estimation, as shown in Figure $5 \mathrm{~b}$ in which saturation occurs at magnitude 6.5. In addition, comparing to $\tau_{c}$ estimation, the scattering of the $P d$ estimation is significantly small.
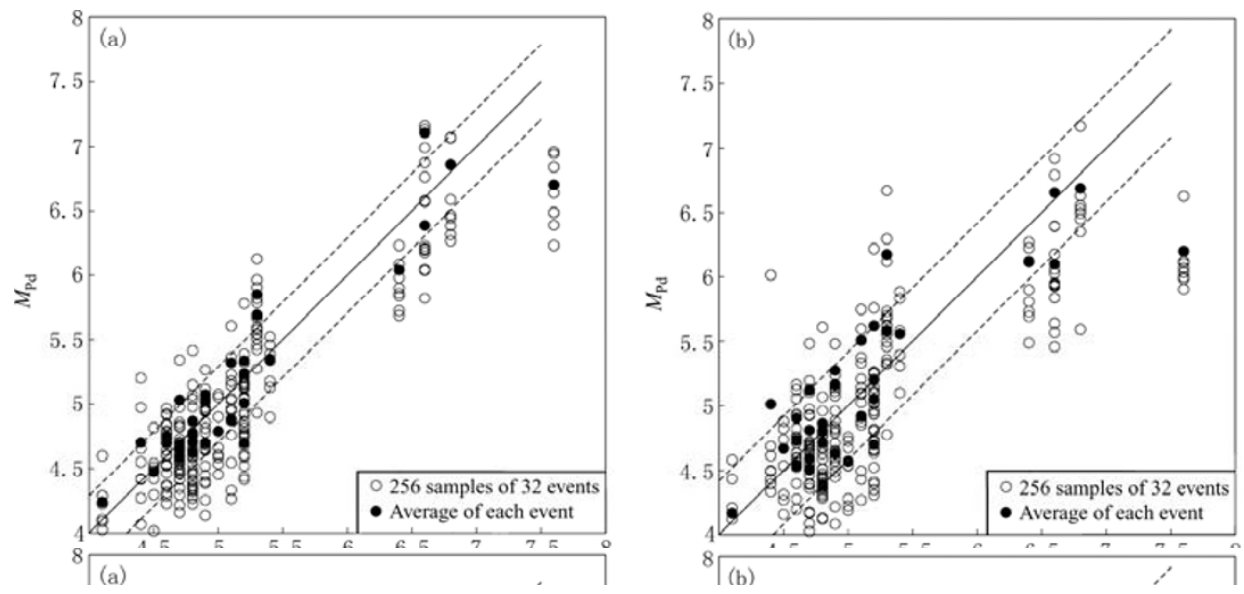

Figure 4 Magnitude estimated from $P d$ calculated using strong motion recordings (a) and using simulated short-period recordings (b) (vertical axis) versus the magnitude of the earthquake (horizontal axis)

Solid line shows the least squares fit and two dashed lines show the range of one standard deviation. In Figure 4a standard deviation is 0.29 and correlation coefficient is 0.93 , and in Figure $4 \mathrm{~b}$ standard deviation is 0.42 and correlation coefficient is 0.85
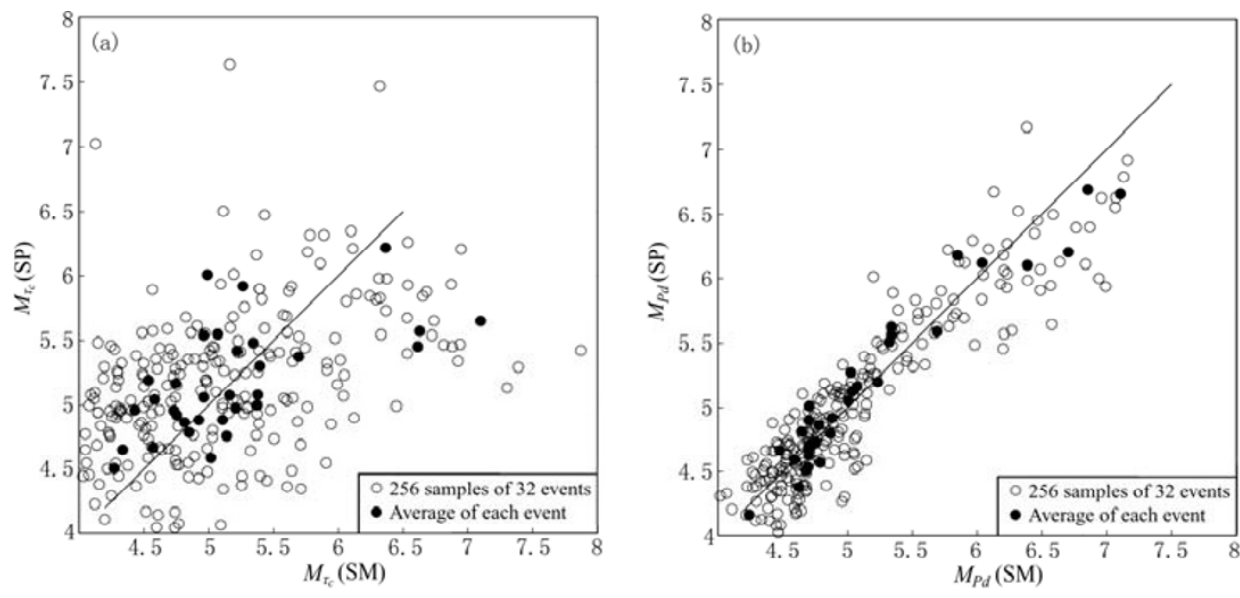

Figure 5 Comparison of the $\tau_{c}$-magnitudes from (broadband) strong motion recordings (horizontal axis) with that from simulated short-period recordings (vertical axis) (a); Comparison of the $P d$-magnitudes from strong motion recordings (horizontal axis) with that from simulated short-period recordings (vertical axis) (b)

The solid line show the theoretical relation $M(\mathrm{SM})=M(\mathrm{SP})$ 


\section{Discussion and conclusions}

Established relations between $\tau_{c}$ or $P d$ and magnitude of earthquakes are basically for broadband recordings. In many places, however, most of the stations deployed are short-period. Therefore it is useful to investigate whether earthquake magnitude can be estimated by short-period recordings with $\tau_{c}$ and/or $P d$ measurements. The result has direct implications for whether short-period seismograph network can be used in earthquake early warning systems.

In this study, we carried out a simulated experiment using the strong motion recordings of the 1999 Chi-Chi earthquake sequence. Not surprisingly short-period recordings may lead to saturation. But the significance of our work is to make clear the magnitude at which saturation starts to occur. For the $\tau_{c}$ estimation, apparent saturation occurs at magnitude 6 . The saturation magnitude is higher for the $P d$ estimation: Apparent saturation occurs at magnitude 6.5. Physical reasons for such a difference can be seen from Figure 2: Comparing the original strong motion acceleration recording with the simulated short-period acceleration recording, difference in amplitudes is smaller than the difference in frequency contents. It can be seen that the strong motion recording has a long-period content (as can be seen more clearly from the portion of seismogram at 7 to $9 \mathrm{~s}$ ), which is eliminated after the high-pass filtering (the convolution of original strong motion recording with the short-period instrument response can be seen as the original recording passes through a high-pass filter) in simulating the short-period recording. Moreover, because $P d$ is the peak displacement amplitude, and the displacement is obtained by twice integration of acceleration records, in the language of broadband/cumulative magnitude (Bormann and Wylegalla, 2005), $P d$ plays the similar role to the $\Sigma m_{\mathrm{B}}$ measured from velocity recordings and thus has less saturation.

The present results suggest that, using the first three seconds of the $\mathrm{P}$ wave seismograms to estimate the size of an earthquake can be applied to short-period recordings to some extent, while the $P d$ estimation seems better than the $\tau_{c}$ estimation with a higher saturation magnitude. In addition, when applying short-period seismograph network in the EEW operation, $P d$ measurement is more preferred than $\tau_{c}$ measurement.

Practically, however, such an observation does not mean that short-period seismograph networks can replace strong motion networks in the earthquake early warning. Combining with broadband instruments, short-period seismographs can accelerate the speed of earthquake report by using the technique of EEWS. They can even play the role of EEWS for intermediate strength events such as those with magnitude 6 . For larger events one has to consider the problem that the amplitude of the strong ground motion exceeds the recording capability provided by the dynamic range of the short-period seismographs, and in some cases destruction of the seismograph itself, or more often the destruction of the communication link. However, even if in the later case, since only the first three seconds of $\mathrm{P}$ wave are used for magnitude estimation, the short-period seismograph networks can still report the message of the strong earthquake before the network stops functioning.

Acknowledgements Thanks are due to Prof. Y.-M. Wu for guidance and helps in developing the earthquake early warning system in North China.

\section{References}

Allen R M and Kanamori H. 2003. The potential for earthquake early warning in Southern California [J]. Science, 300: 786-789, doi: $10.1126 /$ science. 1080912 .

Allen R V. 1978. Automatic earthquake recognition and timing from single traces [J]. Bull Seism Soc Amer, 68: $1521-1532$. 
Boese M, Ionescu C, Wenzel F. 2007. Earthquake early warning for Bucharest, Romania: Novel and revised scalling relations [J]. Geophys Res Lett, 34: L07302, doi:10.1029/2007GL029396.

Bormann P and Wylegalla K. 2005. Quick estimator of the size of great earthquakes [J]. EOS, 86: 464.

Chen Y L, Sammis C G, Teng T L. 2006. A high-frequency view of the 1999 Chi-Chi, Taiwan, source rupture and fault mechanics [J]. Bull Seism Soc Amer, 96(3): 807-820, doi:10.1785/0120050059.

Espinosa-Aranda J M, Jiménez A, Ibarrola G, et al. 1995. Mexico City seismic alert system [J]. Seism Res Lett, 66(6): 42-53.

Heaton T H. 1985. A model for a seismic computerized alert network [J]. Science, 228: 987-990.

Horiuchi S, Negishi H, Abe K, et al. 2005. An automatic processing system for broadcasting earthquake alarms [J]. Bull Seism Soc Amer, 95: 708-718, doi: 10.1785/0120030133.

Institute of Geophysics, China Earthquake Administration. 2006. History of Seismological Observation and Interpretation of the Institute of Geophysics, China Earthquake Administration [M]. Beijing: Seismological Press: 300-334 (in Chinese).

Kanamori H, Hauksson E, Heaton T. 1997. Real-time seismology and earthquake hazard mitigation [J]. Nature, 390: 461-464, doi: $10.1038 / 37280$.

Kanamori H. 2005. Real-time seismology and earthquake damage mitigation [J]. Annu Rev Earth Planet Sci, 33: 195-214, doi: 10.1146/annurev.earth.33.092203.122626.

Lee W H K, Shin T C, Kuo K W, et al. 2001. CWB Free-Field Strong-Motion Data from the 921 Chi-Chi Earthquake: Processed Acceleration Files on CD-ROM [DB]. Taipei: Seismological Observation Center, Central Weather Bureau.

Nakamura Y. 1988. On the urgent earthquake detection and alarm system (UrEDAS) [C]//Proceedings of the 9th World Conference on Earthquake Engineering. Tokyo: [s.n.] VII: 673-678.

Nakamura Y. 1989. Earthquake alarm system for Japan railways [J]. Jpn Railway Eng, 109: 1-7.

Olson E L and Allen R M. 2005. The deterministic nature of earthquake rupture [J]. Nature, 438: 212- 215, doi: 10.1038/nature04214.

Rydelek P and Horiuchi S. 2006. Is earthquake rupture deterministic? [J]. Nature, 442: E5-E6.

Wu C J, Takeo M, Ide S. 2001. Source process of the Chi-Chi earthquake: A joint inversion of strong motion data and global positioning system data with a multifault model [J]. Bull Seism Soc Amer, 91(5): 1 128-1 143.

Wu Y M and Kanamori H. 2005a. Experiment on an onsite early warning method for the Taiwan early warning system [J]. Bull Seism Soc Amer, 95: 347-353, doi: 10.1785/0120040097.

Wu Y M and Kanamori H. 2005b. Rapid assessment of damaging potential of earthquakes in Taiwan from the beginning of P waves [J]. Bull Seism Soc Amer, 95: 1 181-1 185, doi: 10.1785/0120040193.

Wu Y M and Kanamori H. 2007. Exploring the feasibility of on-site earthquake early warning using close-in records of the 2007 Noto Hanto earthquake [J]. Earth Planets and Space, in press.

Wu Y M and Teng T L. 2002. A virtual subnetwork approach to earthquake early warning [J]. Bull Seism Soc Amer, 92: 2 008-2 018, doi: $10.1785 / 0120010217$

Wu Y M and Zhao L. 2006. Magnitude estimation using the first three seconds P-wave amplitude in earthquake early warning [J]. Geophys Res Lett, 33: L16312, doi: 10.1029/2006GL026871.

Wu Y M, Kanamori H, Allen R M, et al. 2007. Determination of earthquake early warning parameters, $\tau_{c}$ and $P d$, for southern California [J]. Geophys J Int, 170: 711-717, doi: 10.1111/j.1365-246X.2007.03430.X.

Wu Y M, Yen H Y, Zhao L, et al. 2006. Magnitude determination using initial P waves: A single-station approach [J]. Geophys Res Lett, 33: L05306, doi: 10.1029/2005GL025395.

Zollo A, Lancieri M, Nielson S. 2006. Earthquake magnitude estimation from peak amplitude of very early seismic signals on strong motion records [J]. Geophys Res Lett, 33: L23312, doi: 10.1029/2006GL027795. 